\title{
A Novel Wireless 3D Localization Method Supported by WSN
}

\author{
http://dx.doi.org/10.3991/ijoe.v9i2.2585 \\ Jianqi Liu, Qinruo Wang, Xiaohu Chen, Wanghua Huang \\ Guangdong University of Technology, Guangzhou, China
}

\begin{abstract}
In many applications, the location information should be necessary. This paper propose a cheap multiplane 3D localization solution, which based on the existing 2D localization system, a perpendicular 2D plane is added to achieve 3D localization. The algorithm is intended for applications which require information of the height, but remain less sensitive to the location accuracy. At the same time, this paper also introduces symmetrical double-sided two-way ranging (SDS-TWR) and dynamic power consumption intensity searching algorithm into localization system, which can effectively lessen the influence of time synchronization and ease internet traffic congestion of wireless localization network.
\end{abstract}

Index Terms-wireless sensor localization; 3D localization; Chirp spread spectrum; dynamic power searching

\section{INTRODUCTION}

Wireless localization systems include base stations localization (like Chirp spread spectrum (CSS) [1], Ultra wideband (UWB) [2], Zigbee [3], WiFi [4], RFID [5, 6], GPS [7], and GSM/CDMA), optical localization technology (like laser scanning, ranging, image recognition) and sonic localization (ultrasonic) [8]. Since optical and sonic systems have backsides like highlycomplex deployment, expensive cost and low system volume [9]. However, wireless localization system boasts easier deployment, convenient maintenance and application into the practical engineering projects [10-12]. Moreover, the localization accuracy ranging from $30 \mathrm{~cm}$ to $50 \mathrm{~cm}$ meets more the practical demands of customers. Among the common wireless localization technologies, RFID or radio frequency identification has developed for a long time and is highly practical with its localization accuracy ranging between 3 to $10 \mathrm{~m}$; Zigbee has good antiinterference and is of low energy consumption with its localization accuracy ranging from 3 to $5 \mathrm{~m}$; and UWB or ultra wide band is of high localization accuracy, which ranges from 0.15 to $1 \mathrm{~m}$. Of all the above mentioned, UWB is of the highest localization accuracy, but its cost is also quite high. It is intended for governments, soldiers and policemen who have a high demand of the accuracy and don't mind about the cost. However, the working frequency of UWB is not yet open. Previously, CSS was mainly applied to pulse compression radar and succeeded in resolving the contradiction that the distance measured and measurement accuracy cannot be optimized simultaneously $[13,14]$. Therefore, research both home and abroad is confined to the field of radar [15-17] In recent years, as IEEE listed CSS as one of the underlying application means of 802.15.4a technology standard, CSS has gradually drawn attention in the field of communications [18].

\section{RESEARCH QUESTION}

In many situations like monitoring of the students in school, correct information of the students' 2D location is called forth. However, about the information of the height, only which store of the building should be informed. Previously, distance model was used to localize:

$c t=d=\sqrt{\left(x-x_{a}\right)^{2}+\left(y-y_{a}\right)^{2}+\left(z-z_{a}\right)^{2}}$

Where, $c$ stands for the speed of electromagnetic wave, about $3 \times 108 \mathrm{~m} / \mathrm{s}$; $t$ for the time that the mobile node Mobiles to the anchor node; $\mathrm{d}$ for the distance between the above mentioned two kinds of nodes; $(x, y, z)$ is the coordinate of the mobile node; while $\left(x_{a}, y_{a}, z_{z}\right)$ is that of the anchor node A or Anchor A. As is shown in Figure 1 , the estimation of the location is worked out by referring to the least square (LS) method or the maximum likelihood (ML) estimation and other algorithms.

However, among the existing localization systems, most have achieved 2D localization, but height information is not included. It costs a great deal to renew the deployment of the localization system. The most feasible method is to refer to the 2D localization system. To obtain the height information without increasing the cost remains an urgent issue to be solved.

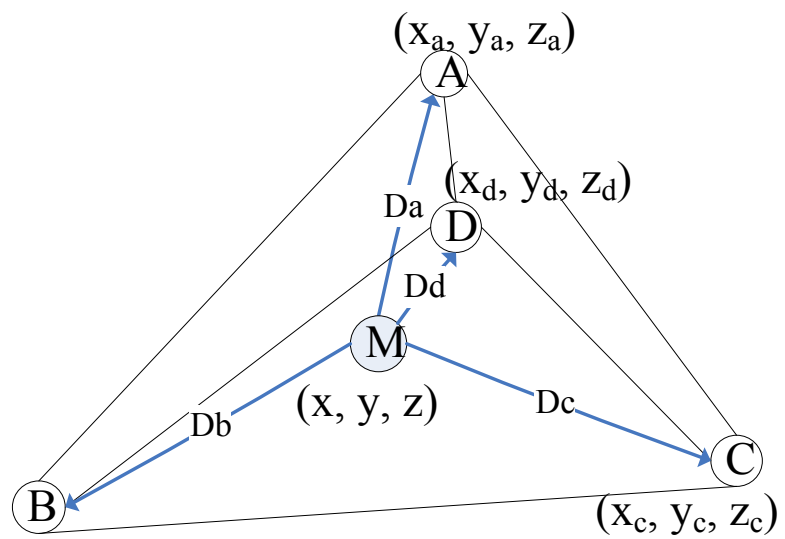

$\left(\mathrm{x}_{\mathrm{b}}, \mathrm{y}_{\mathrm{b}}, \mathrm{z}_{\mathrm{b}}\right)$

Figure 1. Schematic diagram of 3D localization 


\section{Multi-PLANE 3D LOCALIZATION ALGORITHM}

Multi-plane 3D localization technology is one of the range-based localization. It employs SDS-TWR to fulfill the measurement to effectively achieve time synchronization. In order to ease the net communication pressure, especially the influence of the broadcast on the whole internet, dynamic adjustment of the power is utilized to seek the proper anchor node.

\section{A. SDS-TWR}

The distance between Mobile node A and Anchor node $\mathrm{B}$ is estimated through the time electromagnetic wave takes to from A to B. $d$ for distance is the product of electromagnetic wave's transmission time $T_{\text {round }}$ and its transmission speed $c$. If the error is $1 \mathrm{~m}$, the error of $T_{\text {round }}$ can be limited with 3 ns. It costs much to make time synchronization less than 3 ns. However, SDS-TWR has no strict requirement of time synchronization. As is shown in Figure 2, $T_{\text {round }}$ is worked out by the following algorithm. The mobile node sends localization request to the anchor node at $t_{0}$, and the time for the anchor node to receive and handle the request is $t_{p b}$. The mobile node confirms the reception of the signal from the anchor node at $t_{1}$. Thus,

$$
T_{\text {roundA }}=\left(t_{1}-t_{0}-t_{p b}\right) / 2
$$

From (2), $T_{\text {round }}$ can be obtained, in which $t_{0}$ and $t_{0}$ are confirmed at Mobile A. In this method, time synchronization is not a problem, but there induces a new error caused by the difference in the crystal oscillator of the anchor node and the mobile node. That error is small and can be avoided by selecting the crystal oscillator from the same batch of the same manufacturer so as to meet the requirement of the clock.

However, in order to eliminate such errors, after Anchor B confirms its reception of the signal from Mobile node $\mathrm{A}$, it sends to Mobile node $\mathrm{A}$ the range signal at $t_{2}$. After the handling time of $t_{p a}$, Anchor $\mathrm{B}$ confirms the reception of the signal at $t_{3}$. Thus

$$
T_{\text {roundB }}=\left(t_{3}-t_{2}-t_{p a}\right) / 2
$$

From (3), $T_{\text {round }}$ is obtained. And the time costs for Mobile node A to be transmitted to Anchor node B is calculated by (4).

$$
T_{\text {roundB }}=\left(t_{\text {roundA }}+t_{\text {round } B}\right) / 2
$$

In order to lower the cost, the accurate time synchronization is not adopted. At the same, the overall error is effectively controlled within the acceptable scope.

The communication protocol in wireless localization network is as shown in Figure 3. At first Mobile node send a broadcast signal to all anchor nodes by dynamic power searching algorithm addressed in next section. Mobile A regularly sends localization requests to Anchor $\mathrm{B}$, and its localization requests signal are active. At the same time, the sending time $t_{0}$ is recorded. After Anchor

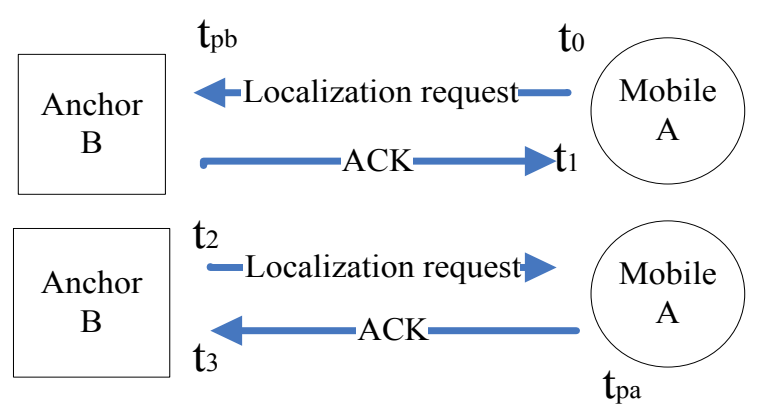

Figure 2. SDS-TWR method

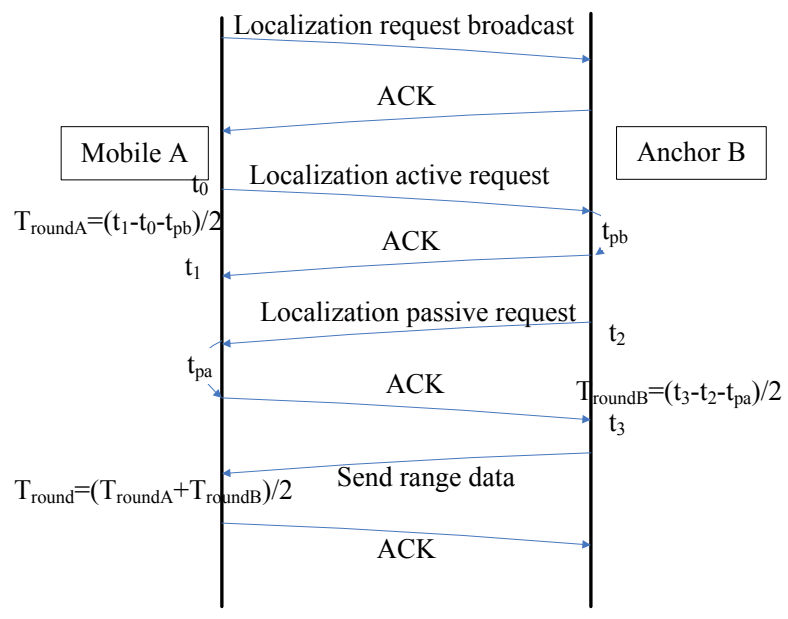

Figure 3. Range localization communication protocol

$B$ receives the localization active request,confirmation data package is generated and sent back. The time delay due to the handling of the signal is $t_{p b}$. Mobile A receives the active request confirmation data package at $t_{1}$. The time delay from Mobile A to Anchor B is $T_{\text {roundA }}$, and $T_{\text {roundB }}$ an be obtained through the similar way. Then Anchor B sends the distance data package $T_{\text {roundB }}$ to Mobile A, and the time delay $T_{\text {round }}$ can be obtained.

\section{B. Dynamic power searching algorithm}

The optimal reference anchor node is chosen through the dynamic power capable of adjustment. Localization requests are sent to all anchor nodes, and collision is caused according to the communication protocol of RTS/CTS. Thereafter, the plenty collision will inflict great burden on wireless internet. How to find the proper anchor node for range is the primary issue of the localization work. Generally speaking, the optimal anchor node is situated nearest to the mobile nodes and which costs the lowest energy consumption in the ranging process. By making use of the adjustability of mobile nodes' power, four to five optimal anchor nodes can be found to achieve localization.

The red point $\mathrm{M}$ is the mobile node. Firstly, the lowest power is employed to send to the anchor node the localization request broadcast. After receiving the signal, the anchor node sends out the confirmation data, which is shown in Figure 4. The red broken circle is the radiation 


\section{A Novel Wireless 3D LOCALIZATION METHOD SUPPORTED BY WSN}

scope. Two anchor nodes are found and recorded as well. The radiating and receiving power is enlarged, and continues to broadcast to the outside. The radiation scope is the blue broken circle, and four anchor nodes are found. By gradually increasing the radiating power, the optimal reference anchor node is found. The sending of the data should be controlled within a certain scope to avoid inflicting influence upon the anchor nodes outside the scope. The workload of the anchor nodes of the whole system is effectively decreased, thus preventing the internet collision.

\section{Multi-plane 3D localization algorithm}

According to the dynamic searching algorithm, the mobile node $\mathrm{M}$ searches four anchor nodes, namely $\mathrm{A}, \mathrm{B}$, $\mathrm{C}$ and $\mathrm{D}$, which are at the same plane. Localization is conducted through SDS-TWR. Thereafter, the coordinate of $\mathrm{M}$ at the plane of $\mathrm{XY}$ can be obtained, which is $\left(x_{1}, y_{1}\right)$. Since it can only be localized within 2D space, we need to introduce the new node E. Node E is at the highest point of the localization space. Then the other two planes apart from XY are formed, namely XZ and YZ. According to SDS-TWR, localization requests are sent to A, B and E nodes. And the coordinate of $\mathrm{M}$ at the plane of $\mathrm{XZ}$ is $\left(x_{2}, z_{1}\right)$. Similarly, the mapping coordinate of $\mathrm{M}$ at the plane of $\mathrm{YZ}$ composed by $\mathrm{C}, \mathrm{D}$ and $\mathrm{E}$, that is $\left(y_{2}, z_{2}\right)$. According to (5), the coordinate of $\mathrm{M}$ at the $3 \mathrm{D}$ space is obtained $(x, y, z)$, which is shown in Figure 5 .

$$
\begin{aligned}
& x=\left(x_{1}+x_{2}\right) / 2 \\
& y=\left(y_{1}+y_{2}\right) / 2 \\
& \mathrm{z}=\left(\mathrm{z}_{1}+\mathrm{z}_{2}\right) / 2
\end{aligned}
$$

\section{CONCLUSION}

In order to meet the 3D localization needs of some application, this paper take the multi-plane localization technologies based on 2D to solve relevant questions related to $3 \mathrm{D}$ localization. In the process of $2 \mathrm{D}$ localization, SDS-TWR is employed to effectively solve the inaccuracy caused by time de-synchronization. At the same time, in the process of dynamic energy consumption searching, the wireless network communication traffic is effectively eased to lower the probability of collision. Based on that, the height of target is obtained by employing the perpendicular plane. The algorithm put forward by this paper is of great application value to situations where height is required.

\section{ACKNOWLEDGMENT}

The authors would like to thank the Natural Science Foundation of Guangdong Province, China (No.9151009001000021, S201101000115 5), the Ministry of Education of Guangdong Province Special Fund Funded Projects through the Cooperative of China (No. 2009B090300 341), the National Natural Science Foundation of China (No. 61262013), and the High-level Talent Project for Universities, Guangdong Province, China (No. 431, YueCaiJiao 2011) for their support in this research.

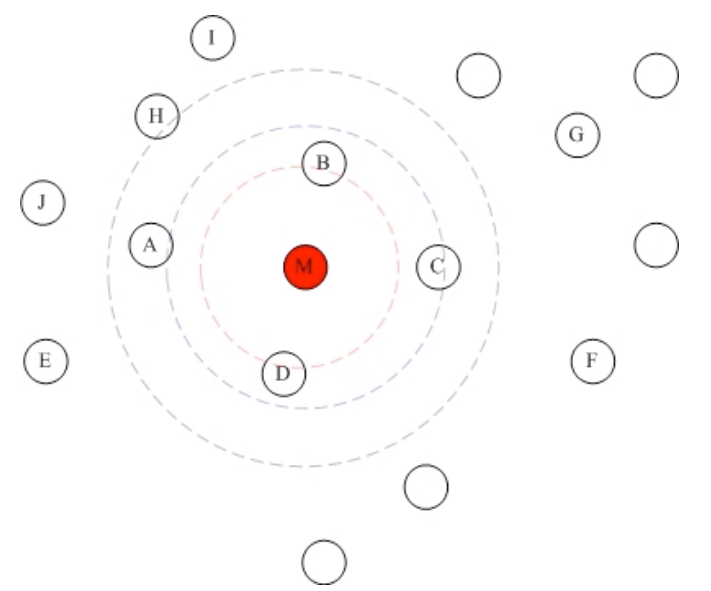

Figure 4. Choosing the optimal reference anchor node

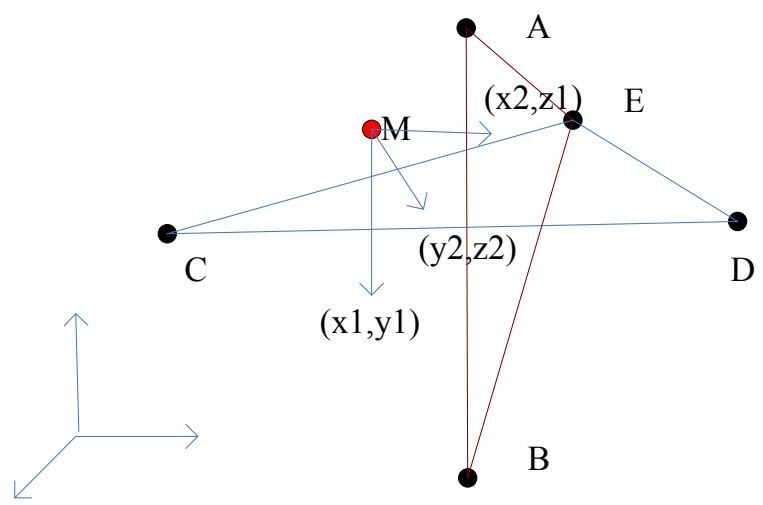

Figure 5. Schematic diagram of the multi-plane wireless localization algorithm

\section{REFERENCES}

[1] Nanotron find. Available: http://www.nanotron.com/EN/PR find. php

[2] ubisense. Available: http://www.ubisense.net/

[3] K. Lorincz and M. Welsh, "MoteTrack: A Robust, Decentralized Approach to RF-Based Location Tracking," in Location- and Context-Awareness. vol. 3479, T. Strang and C. Linnhoff-Popien, Eds., ed: Springer Berlin Heidelberg, 2005, pp. 63-82.

[4] P. A. Zandbergen, "Accuracy of iPhone Locations: A Comparison of Assisted GPS, WiFi and Cellular Positioning," Transactions in GIS, vol. 13, 2009, pp. 5-25. http://dx.doi.org/10.1111/j.14679671.2009.01152.x

[5] M. Chen, et al., "A 2G-RFID-based e-healthcare system," Wireless Communications, IEEE, vol. 17, 2010, pp. 37-43. http://dx.doi.org/10.1109/MWC.2010.5416348

[6] E. A. Fry and L. A. Lenert, "MASCAL: RFID tracking of patients, staff and equipment to enhance hospital response to mass casualty events," AMIA Annual Symposium proceedings 2005, pp. 261265.

[7] R. Bajaj, et al., "GPS: location-tracking technology," Computer, vol. 35, 2002, pp. 92-94.

[8] C. Wang, et al., "Energy Efficient Routing for Multi-hop Underwater Acoustic Networks" International Journal of Computer Science Issues, vol. 9, Nov. 2012.

[9] B. Paramvir and N. P. Ven-kata, "RADAR: An In-Building RFbased User Location and Tracking System," in Proc. of the19th Annual Joint Conf. of the IEEE Computer and Communications Societies, Tel Aviv, 2000, pp. 775-784.

[10] H. Suo, et al., "Issues and Challenges of Wireless Sensor Networks Localization in Emerging Applications," in Proc. of 2012 Int. Conf. on Computer Science and Electronics Engineering, Hongzhou, 2012, pp. 447-451. http://dx.doi.org/10.1109/ ICCSEE.2012.44 


\section{A Novel Wireless 3D LOCALIZATION METHOD SUPPORTED BY WSN}

[11] J. Wan, et al., "Advances in Cyber-Physical Systems Research," KSII Transactions on Internet and Information Systems, vol. 5, 2011, pp. 1891-1908. http://dx.doi.org/10.3837/tiis.2011.11.001

[12] J. Liu, et al., "Towards Real-Time Indoor Localization in Wireless Sensor Networks," in Computer and Information Technology (CIT), 2012 IEEE 12th International Conference on, 2012, pp. 877-884.

[13] G. NGENDAKUMANA and N. E. OUAZZANI, "Ultra WideBand Ultra WideBand Matching of the Rectangular Microstrip Patch Antennas (RMPA) Using Microstrip Non Uniform Transmission Lines ( MNUTL) " International Journal of Computer Science Issues, vol. 9, Nov. 2012.

[14] S. K. Dhar, et al., "3.51pJ/pulse/1.2V CMOS IR-UWB Transmitter " international Journal of Computer Science Issues, vol. 9, Nov. 2012.

[15] J. Wan, et al., "A General Test Platform for Cyber-Physical Systems: Unmanned Vehicle with Wireless Sensor Network Navigation," Procedia Engineering, vol. 24, 2011, pp. 123-127. http://dx.doi.org/10.1016/j.proeng.2011.11.2613

[16] M. Chen, et al., "Machine-to-Machine Communications: architectures, standards, and applications," KSII Transactions on Internet and Information Systems, vol. 6, 2012, pp. 480-497.

[17] M. Chen, et al., "Software agent-based intelligence for codecentric RFID systems," IEEE Intelligent Systems, vol. 25, 2010, pp. 12-19. http://dx.doi.org/10.1109/MIS.2010.44

[18] "IEEE Standard for Information Technology," in Part 802.15.4: Wireless medium access control (MAC) and physical layer (PHY) specifications for low-rate wireless personal area networks ed, 2006.

\section{AUTHORS}

Jianqi Liu is a member of the IEEE, received M.S. degree in computer application technology from the Guangdong University of Technology of China in 2008.
He is currently working toward the Ph.D. degree in the Faculty of Automation with GDUT. His research interests are in the areas of body area network and wireless sensor network.

Qinruo Wang received a B.E. degree from Guangdong University of Technology, China, and a M.A. degree from Zhejiang University, China. He is now with the Faculty of Automation, Guangdong University of Technology, China as a Prof. and an instructor of Ph.D. students. His current research interests include automatic equipment and techniques, mechantronics, and automatic network control.

Xiaohu Chen received B.A. degree in Management Information Systems (MIS) from the Huizhou university of China in 2012. He is currently working toward the M.A. degree in computer application technology from the Guangdong University of Technology of China. His research interests are in the areas of body area network and wireless sensor network.

Wanghua Huang received M.A. degree in computer application technology form the Guangdong University of Technology of China in 2008. He is currently working toward the Ph.D. degree in the Faculty of Automation with GDUT. His research interests are in the areas of pattern recognition and wireless sensor network.

This article is an extended and modified version of a paper presented at the International Conference on Mechanical Engineering, Automation and Material Science (MEAMS2012), held 22-23 December 2012, Wuhan, China. Received 16 March 2013. Published as resubmitted by the authors 25 March 2013. 